\title{
On the Increments of Stable Subordinators
}

\author{
Abdelkader Bahram ${ }^{1,2}$, Bader Almohaimeed ${ }^{2}$ \\ ${ }^{1}$ Department of Mathematics, Djillali Liabes University, Sidi-Bel-Abbes, Algeria \\ ${ }^{2}$ Department of Mathematics, Faculty of Science, Qassim University, Saudi Arabia \\ Email: menaouar_1926@yahoo.fr,bsmhiemied@qu.edu.sa
}

How to cite this paper: Bahram, A. and Almohaimeed, B. (2017) On the Increments of Stable Subordinators. Applied Mathematics, 8, 663-670.

https://doi.org/10.4236/am.2017.85053

Received: April 16, 2017

Accepted: May 20, 2017

Published: May 23, 2017

Copyright (C) 2017 by authors and Scientific Research Publishing Inc. This work is licensed under the Creative Commons Attribution International License (CC BY 4.0).

http://creativecommons.org/licenses/by/4.0/

\section{(c) (†) Open Access}

\section{Abstract}

Let $\{X(t), t \geq 0\}$ be a stable subordinator defined on a probability space $(\Omega, \mathcal{F}, \mathcal{A})$ and let $a_{t}$ for $t>0$ be a non-negative valued function. In this paper, it is shown that under varying conditions on $a_{t}$, there exists a function $\lambda_{\beta}(t)$ such that

$$
\liminf _{t \rightarrow \infty} \frac{\left(X\left(t+a_{T}\right)-X(t)\right)}{\lambda_{\beta}(t)}=1 \text { a.s., }
$$

where $\quad \lambda_{\beta}(t)=\theta_{\alpha} a_{t}^{\frac{1}{\alpha}}\left(\log \frac{t}{a_{t}}+\beta \log \log t+(1-\beta) \log \log a_{t}\right)^{\frac{\alpha-1}{\alpha}}, \quad 0 \leq \beta \leq 1$, $\theta_{\alpha}=(B(\alpha))^{\frac{1-\alpha}{\alpha}}$ and $B(\alpha)=(1-\alpha) \alpha^{\frac{\alpha}{1-\alpha}}\left(\cos \left(\frac{\pi \alpha}{2}\right)\right)^{\frac{1}{\alpha-1}}$.

\section{Keywords}

Increments, Stable Subordinators, Iterated Logarithm Laws

\section{Introduction}

Let $\{X(t), t \geq 0\}$ be a stable ordinator with exponent $\alpha$ with $0<\alpha<1$, defined on a probability space $(\Omega, \mathcal{F}, \mathcal{A})$. Let $a_{t}$ for $t>0$ be a non-negative valued function and $Y(t)=X\left(t+a_{t}\right)-X(t), t>0$. Define

$$
\lambda_{\beta}(t)=\theta_{\alpha} a_{t}^{\frac{1}{\alpha}}\left(\log \frac{t}{a_{t}}+\beta \log \log t+(1-\beta) \log \log a_{t}\right)^{\frac{\alpha-1}{\alpha}},
$$

where $0 \leq \beta \leq 1$,

$$
\theta_{\alpha}=(B(\alpha))^{\frac{1-\alpha}{\alpha}} \text { and } B(\alpha)=(1-\alpha) \alpha^{\frac{\alpha}{1-\alpha}}\left(\cos \left(\frac{\pi \alpha}{2}\right)\right)^{\frac{1}{\alpha-1}} \text {. }
$$


For any value of $t$, the characteristic function of $X(t)$ is of the form

$$
E\left(\mathrm{e}^{i u X(t)}\right)=\exp \left(-t|u|^{\alpha}\left(1-\frac{u i}{|u|} \tan \left(\frac{\pi \alpha}{2}\right)\right)\right), \quad 0<\alpha<1 .
$$

Limit theorems on the increments of stable subordinators have been investigated in various directions by many authors [1]-[6]. Among the above many results, we are interested in Fristedt [4] and Vasudeva and Divanji [3] whose results are the following limit theorems on the increments of stable subordinators.

Theorem $1([4])$

$$
\liminf _{t \rightarrow \infty} \theta_{\alpha} t^{-\frac{1}{\alpha}}(\log \log t)^{\frac{1-\alpha}{\alpha}} X(t)=1 \quad \text { almost surely } \quad(\text { a.s }) .
$$

Theorem 2 ([3]) Let $0<a_{t}$ for $t>0$, be a non-decreasing function of $t$ such that

(i) $0<a_{t} \leq t$ for $t>0$,

(ii) $a_{t} \rightarrow \infty$ as $t \rightarrow \infty$, and

(iii) $a_{t} / t$ is non-increasing. Then

$$
\liminf _{t \rightarrow \infty} \frac{\left(X\left(t+a_{t}\right)-X(t)\right)}{\xi(t)}=1 \text { a.s., }
$$

where $\xi(t)=\theta_{\alpha} a_{t}^{\frac{1}{\alpha}}\left(\log \frac{t}{a_{t}}+\log \log t\right)^{\frac{\alpha-1}{\alpha}}$.

In this paper, our aim is to investigate Liminf behaviors of the increments of $Y$. We establish that, under certain conditions on $a_{t}$,

$$
\begin{aligned}
& \liminf _{t \rightarrow \infty} \frac{Y(t)}{\lambda_{\beta}(t)}=1 \quad \text { a.s., } \\
& \text { where } Y(t)=X\left(t+a_{t}\right)-X(t) .
\end{aligned}
$$

Throughout the paper $c$ and $k$ (integer), with or without suffix, stand for positive constants. i.o. means infinitely often. We shall define for each $u \geq 0$ the functions $\log u=\log (\max (u, 1))$ and $\log \log u=\log \log (\max (u, 3))$.

\section{Main Result}

In this section, we reformulate the result obtained in Theorem 2 and establish our main result using $\lambda_{\beta}(t)$ with $0 \leq \beta \leq 1$ instead of $\xi(t)$.

Theorem 3 Let $a_{t}, t>0$, be a non-decreasing function of $t$ such that

(i) $0<a_{t} \leq t$ for $t>0$,

(ii) $a_{t} \rightarrow \infty$ as $t \rightarrow \infty$, and

(iii) $a_{t} / t$ is non-increasing. Then

$$
\liminf _{t \rightarrow \infty} \frac{Y(t)}{\lambda_{\beta}(t)}=1 \text { a.s. }
$$

Remark 1 Let us mention some particular cases

1. For $a_{t}=t$ we obtain Fristedt's iterated logarithm laws (see Thorem 1). 
2. If $\beta=1$ we have Vasudeva and Divanji theorem (see Theorem 2).

3. If $\beta=0$ under assumptions (i), (ii) and (iii) of Theorem 3 we also have

$$
\liminf _{t \rightarrow \infty} \frac{Y(t)}{\lambda_{0}(t)}=1 \text { a.s. }
$$

In order to prove Theorem 3, we need the following Lemma

Lemma 1 (see [3] or [7]) Let $X_{1}$ be a positive stable random variable with characteristic function

$$
E\left(\exp \left\{i u X_{1}\right\}\right)=\exp \left\{-|u|^{\alpha}\left(1-\frac{i u}{|u|} \tan \left(\frac{\pi \alpha}{2}\right)\right)\right\}, 0<\alpha<1 .
$$

Then, as $x \rightarrow 0$,

$$
P\left(X_{1} \leq x\right) \simeq \frac{x^{\frac{\alpha}{2(1-\alpha)}}}{\sqrt{2 \pi \alpha B(\alpha)}} \exp \left\{-B(\alpha) x^{\frac{\alpha}{\alpha-1}}\right\}
$$

where

$$
B(\alpha)=(1-\alpha) \alpha^{\frac{\alpha-1}{\alpha}}\left(\cos \left(\frac{\pi \alpha}{2}\right)\right)^{\frac{1}{\alpha-1}}
$$

Proof of Theorem 3. Firstly, we show that for any given $\varepsilon>0$, as $t \rightarrow \infty$,

$$
P\left(Y(t) \leq(1+\varepsilon) \lambda_{\beta}(t) \text { i.o }\right)=1 \text {. }
$$

Let $u_{1}$ be a number such that $a_{u_{1}}>1$. Define a sequence $\left(u_{k}\right)$ through $u_{k+1}=u_{k}+a_{u_{k}}$, for $k=1,2, \cdots$. Now we show that

$$
P\left(Y\left(u_{k}\right) \leq(1+\varepsilon) \lambda_{\beta}\left(u_{k}\right) \text { i.o }\right)=1 .
$$

From Mijhneer [8], we have

$$
P\left(Y\left(u_{k}\right) \leq(1+\varepsilon) \lambda_{\beta}\left(u_{k}\right)\right)=P\left(X(1) \leq \frac{(1+\varepsilon) \lambda_{\beta}\left(u_{k}\right)}{a_{u_{k}}^{\frac{1}{\alpha}}}\right) .
$$

But

$$
\frac{\lambda_{\beta}\left(u_{k}\right)}{a_{u_{k}}^{\frac{1}{\alpha}}}=\theta_{\alpha}\left(\log \frac{u_{k}}{a_{u_{k}}}+\beta \log \log u_{k}+(1-\beta) \log \log a_{u_{k}}\right)^{\frac{\alpha-1}{\alpha}} .
$$

Applying Lemma 1 with

$$
x=(1+\varepsilon) \theta_{\alpha}\left(\log \frac{u_{k}}{a_{u_{k}}}+\beta \log \log u_{k}+(1-\beta) \log \log a_{u_{k}}\right)^{\frac{\alpha-1}{\alpha}},
$$

one can find a $k_{0}$ such that, for all $k \geq k_{0}$, 


$$
\begin{aligned}
& P\left(X(1) \leq \frac{(1+\varepsilon) \lambda_{\beta}\left(u_{k}\right)}{a_{u_{k}}^{\frac{1}{\alpha}}}\right) \\
& \geq \frac{c_{0}}{2\left(\log \left(\frac{u_{k}\left(\log u_{k}\right)^{\beta}\left(\log a_{u_{k}}\right)^{1-\beta}}{a_{u_{k}}}\right)\right)^{1 / 2}} \\
& \quad \times \exp \left\{-(1+\varepsilon)^{\alpha /(\alpha-1)} \log \left(\frac{u_{k}\left(\log u_{k}\right)^{\beta}\left(\log a_{u_{k}}\right)^{1-\beta}}{a_{u_{k}}}\right)\right\},
\end{aligned}
$$

where $c_{0}$ is some positive constant. Notice that

$$
(1+\varepsilon)^{\frac{\alpha}{\alpha-1}}=\left(1-\varepsilon_{1}\right)<1 \text { for some } \varepsilon_{1}>0 .
$$

Hence

$$
\begin{aligned}
& P\left(X(1) \leq \frac{(1+\varepsilon) \lambda_{\beta}\left(u_{k}\right)}{a_{u_{k}}^{\frac{1}{\alpha}}}\right) \\
& \geq \frac{c_{0}}{\left(\log \left(\frac{u_{k}\left(\log u_{k}\right)^{\beta}\left(\log a_{u_{k}}\right)^{1-\beta}}{a_{u_{k}}}\right)\right)^{1 / 2}\left(\frac{a_{u_{k}}}{u_{k}}\right)} \\
& \times\left(\frac{u_{k}}{a_{u_{k}}}\right)^{\varepsilon_{1}} \frac{1}{\left(\left(\log u_{k}\right)^{\beta}\left(\log a_{u_{k}}\right)^{1-\beta}\right)^{\left(1-\varepsilon_{1}\right)}} \\
& =\frac{c_{0}}{\left(\log \left(\frac{u_{k}\left(\log u_{k}\right)^{\beta}\left(\log a_{u_{k}}\right)^{1-\beta}}{a_{u_{k}}}\right)\right)^{1 / 2}}\left(\frac{u_{k+1}-u_{k}}{u_{k}}\right) \\
& \times\left(\frac{u_{k}}{a_{u_{k}}}\right)^{\varepsilon_{1}} \frac{1}{\left(\left(\log u_{k}\right)^{\beta}\left(\log a_{u_{k}}\right)^{1-\beta}\right)^{\left(1-\varepsilon_{1}\right)}} .
\end{aligned}
$$

Let $1_{k}=u_{k} / a_{u_{k}}$ and $m_{k}=\left(\log u_{k}\right)^{\beta}\left(\log a_{u_{k}}\right)^{1-\beta}$. Note that $1_{k}$ is non-decreasing and $m_{k} \rightarrow \infty$ as $k \rightarrow \infty$. In turn one finds a $k_{1} \geq k_{0}$, such that

$$
\frac{1_{k}^{\varepsilon_{1}} m_{k}^{\varepsilon_{1}}}{\left(\log 1_{k} m_{k}\right)^{1 / 2}} \geq 1 \text {, whenever } k \geq k_{1} \text {. }
$$

Therefore, for all $k \geq k_{1}$, we have 


$$
\begin{aligned}
& P\left(X(1) \leq \frac{(1+\varepsilon) \lambda_{\beta}\left(u_{k}\right)}{a_{u_{k}}^{\frac{1}{\alpha}}}\right) \\
& \geq c_{0} \frac{\left(u_{k+1}-u_{k}\right)}{2 u_{k}\left(\log u_{k}\right)^{\beta}\left(\log a_{u_{k}}\right)^{1-\beta}}=c_{0} \frac{\left(u_{k+1}-u_{k}\right)}{2 u_{k}}\left(\frac{\log a_{u_{k}}}{\log u_{k}}\right)^{\beta} \frac{1}{\log a_{u_{k}}} \\
& \geq c_{0} \frac{\left(u_{k+1}-u_{k}\right)}{2 u_{k}}\left(\frac{\log a_{u_{k}}}{\log u_{k}}\right) \frac{1}{\log a_{u_{k}}}=c_{0} \frac{\left(u_{k+1}-u_{k}\right)}{2 u_{k} \log u_{k}} .
\end{aligned}
$$

Observe that

$$
\int_{k_{1}}^{\infty} \frac{\mathrm{d} t}{t \log t} \leq \sum_{k=k_{1}}^{\infty} \frac{\left(u_{k+1}-u_{k}\right)}{u_{k} \log u_{k}} .
$$

From the fact that $\int_{k_{1}}^{\infty} \frac{\mathrm{d} t}{t \log t}=\infty$ and from (4), (5), and (6) one gets

$$
\sum_{k=1}^{\infty} P\left(Y\left(u_{k}\right) \leq(1+\varepsilon) \lambda_{\beta}\left(u_{k}\right)\right)=\infty .
$$

Observe that $\left(Y\left(u_{k}\right)\right)$ is a sequence of mutually independent random variables (for, $u_{k+1}=u_{k}+a_{u_{k}}$ ) and by applying Borel-Cantelli lemma, we get

$$
P\left(Y\left(u_{k}\right) \leq(1+\varepsilon) \lambda_{\beta}\left(u_{k}\right) \text { i.o }\right)=1
$$

which establishes (3).

Now we complete the proof by showing that, for any $\varepsilon \in(0,1)$,

$$
P\left(Y(t) \leq(1-\varepsilon) \lambda_{\beta}\left(t_{k}\right) \quad \text { i.o }\right)=0 .
$$

Define a subsequence $\left(t_{k}\right)$, such that

$$
a_{t_{k}}=\left(t_{k+1}-t_{k}\right) /\left(\log t_{k}\right)^{(1-\beta)(1+\varepsilon)}, k=1,2, \cdots
$$

and the events $A_{t}$ and $B_{k}$ as

$$
A_{t}=\left\{Y(t) \leq(1-\varepsilon) \lambda_{\beta}(t)\right\}
$$

and

$$
B_{k}=\left\{\inf _{t_{k} \leq t \leq t_{k+1}} Y(t) \leq(1-\varepsilon) \lambda_{\beta}\left(t_{k+1}\right)\right\}, \quad k=1,2, \cdots
$$

Note that

$$
\left(A_{t} \quad \text { i.o., } t \rightarrow \infty\right) \subset\left(B_{k} \quad \text { i.o. }, k \rightarrow \infty\right) .
$$

Further, define

$$
C_{k}=\left\{X\left(t_{k}+a_{t_{k}}\right)-X\left(t_{k+1}\right) \leq(1-\varepsilon) \lambda_{\beta}\left(t_{k+1}\right)\right\}
$$

and observe that

$$
\left(B_{k} \quad \text { i.o., } k \rightarrow \infty\right) \subset\left(C_{k} \quad \text { i.o., } k \rightarrow \infty\right) .
$$

Hence in order to prove (7) it is enough to show that

$$
P\left(C_{k} \quad \text { i.o. }\right)=0 \text {. }
$$

We have 


$$
P\left(X\left(t_{k}+a_{t_{k}}\right)-X\left(t_{k+1}\right) \leq(1-\varepsilon) \lambda_{\beta}\left(t_{k+1}\right)\right)=P\left(X(1) \leq \frac{(1-\varepsilon) \lambda_{\beta}\left(t_{k+1}\right)}{\left(a_{t_{k}}+t_{k}-t_{k+1}\right)^{1 / \alpha}}\right)
$$

and

$$
\begin{aligned}
& \frac{(1-\varepsilon) \lambda_{\beta}\left(t_{k+1}\right)}{\left(a_{t_{k}}+t_{k}-t_{k+1}\right)^{1 / \alpha}} \\
& \simeq(1-\varepsilon) \theta_{\alpha}\left(\frac{a_{t_{k+1}}}{a_{t_{k}}}\right)^{1 / \alpha}\left(\log \left(\frac{t_{k+1}\left(\log t_{k+1}\right)^{\beta}\left(\log a_{t_{k}}\right)^{1-\beta}}{a_{t_{k}}}\right)\right)^{(\alpha-1) / \alpha} .
\end{aligned}
$$

The fact that $a_{t} / t$ is non-increasing as $t \rightarrow \infty$ implies that

$$
\frac{a_{t_{k+1}}}{t_{k+1}} \leq \frac{a_{t_{k}}}{t_{k}} \quad \text { or } \quad \frac{a_{t_{k+1}}}{a_{t_{k}}} \leq \frac{t_{k+1}}{t_{k}} .
$$

Hence for a given $\varepsilon_{1}>0$ satisfying $(1-\varepsilon)\left(1+\varepsilon_{1}\right)^{1 / \alpha}<1$, there exists a $k_{2}$ such that

$$
a_{t_{k+1}} / a_{t_{k}} \leq\left(1+\varepsilon_{1}\right) \text {, for all } k \geq k_{2} .
$$

Let $(1-\varepsilon))\left(1+\varepsilon_{1}\right)^{1 / \alpha}=\left(1-\varepsilon_{2}\right)$. Then, for $k \geq k_{2}$,

$$
P\left(C_{k}\right) \leq P\left(X(1) \leq\left(1-\varepsilon_{2}\right) \theta_{\alpha}\left(\log \frac{t_{k+1}}{a_{t_{k+1}}}\left(\log t_{k+1}\right)^{\beta}\left(\log a_{t_{k+1}}\right)^{1-\beta}\right)^{(\alpha-1) / \alpha}\right) .
$$

From lemma 1, we can find a $k_{3}\left(\geq k_{2}\right)$ such that for all $k \geq k_{3}$,

$$
\begin{aligned}
P\left(C_{k}\right) \leq & c_{1}\left(\log \frac{t_{k+1}}{a_{t_{k+1}}}\left(\log t_{k+1}\right)^{\beta}\left(\log a_{t_{k+1}}\right)^{1-\beta}\right)^{-\frac{1}{2}} \\
& \times \exp \left\{\left(1-\varepsilon_{2}\right)^{\alpha /(\alpha-1)}\left(\log \frac{t_{k+1}}{a_{t_{k}}}\left(\log t_{k+1}\right)^{\beta}\left(\log a_{t_{k+1}}\right)^{1-\beta}\right)\right\},
\end{aligned}
$$

where $c_{1}$ is a positive constant.

Let $\left(1-\varepsilon_{2}\right)^{\alpha /(\alpha-1)}=\left(1+\varepsilon_{3}\right), \quad \varepsilon_{3}>0$. Then, for all $k \geq k_{3}$,

$$
\begin{gathered}
P\left(C_{k}\right) \leq c_{1}\left(\log \frac{t_{k+1}}{a_{t_{k}}}\left(\log t_{k+1}\right)^{\beta}\left(\log a_{t_{k+1}}\right)^{1-\beta}\right)^{-1 / 2}\left(\frac{a_{t_{k+1}}}{t_{k}}\right)^{\left(1+\varepsilon_{3}\right)} \\
\left(\left(\log t_{k+1}\right)^{\beta}\left(\log a_{t_{k+1}}\right)^{1-\beta}\right)^{-\left(1+\varepsilon_{3}\right)} .
\end{gathered}
$$

Since

$$
\left(a_{t_{k+1}} / t_{k+1}\right)^{\left(1+\varepsilon_{3}\right)} \leq\left(a_{t_{k}} / t_{k}\right)^{\left(1+\varepsilon_{3}\right)} \leq a_{t_{k}} / t_{k},
$$

then from (8) and for all $k \geq k_{3}$, we have

$$
P\left(C_{k}\right) \leq c_{1}\left(\log \frac{t_{k}}{a_{t_{k}}}\left(\log t_{k}\right)^{\beta}\left(\log a_{t_{k}}\right)^{1-\beta}\right)^{-1 / 2}\left(\frac{a_{t_{k}}}{t_{k}}\right)\left(\left(\log t_{k}\right)^{\beta}\left(\log a_{t_{k}}\right)^{1-\beta}\right)^{-\left(1+\varepsilon_{3}\right)} .
$$




$$
\begin{aligned}
P\left(C_{k}\right) \leq & C_{1}\left(\log \frac{t_{k}}{a_{t_{k}}}\left(\log t_{k}\right)^{\beta}\left(\log a_{t_{k}}\right)^{1-\beta}\right)^{-1 / 2}\left(\frac{t_{k+1}-t_{k}}{t_{k}}\right) \\
& \times \frac{1}{\left(\log t_{k}\right)^{1+\varepsilon_{3}}} \frac{1}{\left(\log a_{t_{k+1}}\right)^{(1-\beta)\left(1+\varepsilon_{3}\right)}} \\
\leq & c_{1}\left(\frac{t_{k+1}-t_{k}}{t_{k}}\right) \frac{1}{\left(\log t_{k}\right)^{\left(1+\varepsilon_{3}\right)}} .
\end{aligned}
$$

Observe that

$$
\int_{k_{4}}^{\infty} \frac{\mathrm{d} t}{t(\log t)^{\left(1+\varepsilon_{3}\right)}} \geq \sum_{k=k_{4}}^{\infty} \frac{\left(t_{k+1}-t_{k}\right)}{t_{k+1}\left(\log t_{k+1}\right)^{\left(1+\varepsilon_{3}\right)}}
$$

and

$$
\frac{\left(t_{k+1}-t_{k}\right)}{t_{k+1}\left(\log t_{k+1}\right)^{\left(1+\varepsilon_{3}\right)}} \simeq \frac{\left(t_{k+1}-t_{k}\right)}{t_{k}\left(\log t_{k}\right)^{\left(1+\varepsilon_{3}\right)}}
$$

Hence

$$
\sum_{k=k_{4}}^{\infty} \frac{\left(t_{k+1}-t_{k}\right)}{t_{k}\left(\log t_{k}\right)^{\left(1+\varepsilon_{3}\right)}}<\infty
$$

Now we get $\sum_{k=k_{4}}^{\infty} P\left(C_{k}\right)<\infty$, which in turn establishes (9) by applying to the Borel-Cantelli lemma. The proof of Theorem 3 is complete.

\section{Conclusion}

In this paper, we developed some limit theorems on increments of stable subordinators. We reformulated the result obtained by Vasudeva and Divanji [3], and established our result by using $\lambda_{\beta}(t)$.

\section{Acknowledgments}

Our thanks to the experts who have contributed towards development of our paper.

\section{References}

[1] Bahram, A. and Almohaimeed, B. (2016) Some Liminf Results for the Increments of Stable Subordinators. Theoretical Mathematics and Applications, 28, 117-124.

[2] Hawkes, J.A. (1971) Lower Lipschitz Condition for Stable Subordinator. Zeitschrift für Wahrscheinlichkeitstheorie und Verwandte Gebiete, 17, 23-32. https://doi.org/10.1007/BF00538471

[3] Vasudeva, R and Divanji, G. (1988) Law of Iterated Logarithm for Increments of Stable Subordinators. Stochastic Processes and Their Applications, 28, 293-300.

[4] Fristedt, B. (1964) The Behaviour of Increasing Stable Process for Both Small and Large Times. Journal of Applied Mathematics and Mechanics, 13, 849-856.

[5] Fristedt, B and Pruit, W.E. (1971) Lower Functions of Increasing Random Walks and Subordinators. Zeitschrift für Wahrscheinlichkeitstheorie und Verwandte Gebiete, 18, 167-182. https://doi.org/10.1007/BF00563135

[6] Fristedt, B and Pruit, W.E. (1972) Uniform Lower Functions for Subordinators. 
Zeitschrift für Wahrscheinlichkeitstheorie und Verwandte Gebiete, 24, 63-70. https://doi.org/10.1007/BF00532463

[7] Mijhneer, J. L. (1975) Sample Path Properties of Stable Process. Mathematisch Centrum, Amsterdam.

[8] Mijhneer, J.L. (1995) On the Law of Iterated Logarithm for Subsequences for a Stable Subordinator. Journal of Mathematical Sciences, 76, 2283-2286.

https://doi.org/10.1007/BF02362699

Submit or recommend next manuscript to SCIRP and we will provide best service for you:

Accepting pre-submission inquiries through Email, Facebook, LinkedIn, Twitter, etc. A wide selection of journals (inclusive of 9 subjects, more than 200 journals)

Providing 24-hour high-quality service

User-friendly online submission system

Fair and swift peer-review system

Efficient typesetting and proofreading procedure

Display of the result of downloads and visits, as well as the number of cited articles Maximum dissemination of your research work

Submit your manuscript at: http://papersubmission.scirp.org/

Or contact am@scirp.org 